\title{
Modelling and Control of Quadrotor Control System using MATLAB/Simulink
}

\author{
Wai Mar Thet \\ Department of Electronics \\ Engineering \\ Technological University \\ Thanlyin, Myanmar
}

\author{
Myo Myint \\ Department of Electronics \\ Engineering \\ Technological University \\ Thanlyin, Myanmar
}

\author{
Ei Ei Khin \\ Department of Electronics \\ Engineering \\ Technological University \\ Thanlyin, Myanmar
}

\begin{abstract}
Generally, Quadrotor type Unmanned Aerial Vehicles are unstable in nature, so to stabilize it, the controller is used. This paper observes the PD controller that make use of UAV to control the adjust of quadrotor UAV even as in the air. The gain parameters of the PD controller, the proportional gain $\mathrm{K}_{\mathrm{p}}$ and the derivative gain $\mathrm{K}_{\mathrm{d}}$ are apply to be stable and good performance. Unmanned aerial vehicles (UAV) are becoming increasingly common and span a huge range of size and shape. After integrating PD controllers into the systems, quadcopter settling time of roll, pitch and yaw system. Simulations result and comparison of X, Yand Yaw control techniques are presented at the end of this paper. This controller monitors the controlled process variable, and compares it with the reference or set point. The difference between actual and desired value of the process variable, called the error signal. Error is applied as feedback to generate a control action to bring the controlled process variable to the same value as the set point. Control action in which the output is proportional to a linear combination of the input and the time rate of change of input.
\end{abstract}

Keywords: stabilization; integrating; simulations result and comparison; error signal; control action

\section{INTRODUCTION}

UAVs or 'Unmanned Aerial Vehicles' are defined as aircrafts without the onboard presence of pilot. Research and development of unmanned aerial vehicle (UAV) are getting high encouragement nowadays, since the application of UAV can apply to variety of area such as rescue mission, military, film making, agriculture and others. For search and rescue mission, UAV that attached with infrared cameras assist the mission to search the target. Quadcopter or quadrotor aircraft is one of the UAV that is major focuses of active researches in recent years. Quadcopter operated by thrust that produce by four motors that attached to it body. It has four input force and six output states $(\mathrm{x}, \mathrm{y}, \mathrm{z}, \theta, \psi, \omega)$ and it is an under-actuated system, Quadcopter has the advantages over the conventional helicopter where the mechanical design is simpler. UAVs are usually used for activities which are deemed to be either too dull, dirty or dangerous for humans, and as a result, eliminates the risk of loss of human life for certain applications [1]. UAVs can be remotely controlled or flown autonomously based on pre-programmed flight plans. Hence, plenty of research has been done with regards to control techniques for the trajectory tracking of the flight plans. Quadrotors are now widely available, both as commercial products and as opensource projects. Usually UAV systems are unstable and stabilization control plays a very important role in it. It automatically stabilizes the attitude of the vehicle and prevents the vehicle from flopping and also maintains the desired orientation during flight. The Quadrotor UAV stabilization is depend upon the appropriate controlling of their four motor speed. A stable flight is necessary for safety and for better flight experience and to achieve a successful mission. The four rotors are used in controlling the vehicle. Each rotor produces moments as well as vertical forces. These moments have been experimentally observed. The flight movement of quad-rotor UAV is controlled by varying the speed of each propeller and the attitude stabilization can be achieved by controlling the required speed of each motor. UAVs can be remotely controlled or flown autonomously based on pre-programmed flight plans. Hence, plenty of research has been done with regards to control techniques for the trajectory tracking of the flight plans. Nowadays, one of the most popular UAV type is the rotary fixed-wing UAV. They are highly lauded due to the advantages they have, such as; vertical take-off and landing ability, portability, good mobility, ability to carry payload, and also the ability to operate in constraint spaces. The main contribution of this paper is to introduce a new based PD control algorithm for attitude stabilization of Quad-rotor UAV. It is worth to mention that the proposed algorithm was implemented and tested on developed Quadrotor UAV system and compared with conventional PD Controller.

\section{MODELLING OF A QUADROTOR}

\subsection{Aerodynamics Forces and Torques}

Quadrotor needs a mechanism for generating forces and torques that are required to control its horizontal and vertical movements. There are four main forces that exert on a quadrotor: gravity, lift, thrust and drag. Gravity is a force that pulls the quadrotor down because of its mass. Lift and thrust are the upward reaction forces acting on quadrotor due to the propellers. Finally, drag is the backward force on the quadrotor due to air. Quadrotor mechanism is mainly based on its rotors and propellers that generate thrust perpendicular to its rotor. The main thrust is generated along $\mathrm{Z}$ axis that creates vertical movement. The horizontal movements along $\mathrm{X}$ and $\mathrm{Y}$ axes are resulted from directing the force or thrust vector in the appropriate direction. Therefore, quadrotor can be characterized by one main control force $\mathrm{T} b \mathrm{uF}$, collective lift $\mathrm{u}$ is the sum of the thrust generated by the four propellers. 
Where 1 is distance from the propellers to the center of mass of the quadrotor and $\mathrm{Q}$ is the fan torques due to air.

The collective lift $\mathrm{u}$ is the sum of the thrust generated by the four propellers.

$$
\alpha=\sum_{i=1}^{4} f_{i}
$$

Torque produced by each axis is resulted from

$$
\begin{aligned}
& Y_{\theta}=b\left(f_{2}-f_{4}\right) \\
& Y_{\varphi}=b\left(f_{3}-f_{1}\right) \\
& Y_{\varphi}=Q_{1}+Q_{2}+Q_{3}+Q_{4}
\end{aligned}
$$

Where $\mathrm{b}$ is distance from the propellers to the center of mass of the quadrotor and $\mathrm{Q}$ is the fan torques due to air drag. $\theta^{\circ}{ }_{\text {roll }}, \theta^{\circ}$ pitch and $\theta^{\circ}$ yaw are the roll, pitch and yaw angular velocity that can be received from gyro sensor. The error values will be

$$
\begin{aligned}
& e_{\text {roll }}=\theta_{\text {rollde }}^{\circ}-\theta_{\text {roll }}^{\circ} \\
& e_{\text {pitch }}=\theta_{\text {pitchde }}^{\circ}-\theta_{\text {pitch }}^{\circ} \\
& e_{\text {yaw }}=\theta_{\text {yawde }}^{\circ}-\theta_{\text {yaw }}^{\circ}
\end{aligned}
$$

where rollde $\theta^{\circ}{ }_{\text {rollde }}$, pitchde $\theta^{\circ}$ pitchde and yawde $\theta^{\circ}$ yawde represent desired angular velocity. Using these error values in $\mathrm{PD}$ equation, it can be gained

$$
\begin{aligned}
& \mu_{\text {roll }}=k_{p} e_{\text {roll }}+k_{d} \frac{d e_{\text {roll }}}{d t} \\
& \mu_{\text {pitch }}=k_{p} e_{\text {pitch }}+k_{d} \frac{d e_{\text {piteh }}}{d t} \\
& \mu_{\text {yaw }}=k_{p} e_{\text {yaw }}+k_{d} \frac{d e_{\text {yaw }}}{d t}
\end{aligned}
$$

\subsection{Quadrotor Configuration}

Quadrotor UAV can be assigned to two different configurations; plus, and cross configuration. In this case, four brushless DC motors are mounted on quadrotor UAV in cross configuration. One pair of motors $(1,3)$ rotates in counter clockwise direction while the other pair of motors $(2,4)$ rotates in clockwise direction as shown in fig. The motion of quadrotor is achieved by varying the motor speed. Thus, increasing or decreasing the four motor's speeds together generates vertical motion. Increasing motor $(1,2)$ speed or motor $(3,4)$ speed produces roll rotation that results quadrotor to bend left or bend right. The same method is used for pitch control. Varying motors $(1,4)$ and motors $(2,3)$ speed conversely produce pitch rotation that results quadrotor to go forward or backward. Yaw rotation can be done by the difference in the counter-torque between each pair of motors.

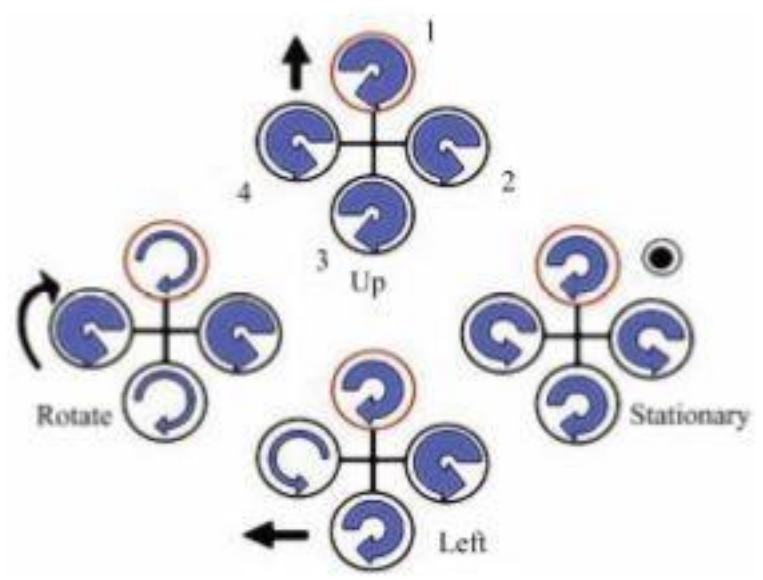

Figure 1. Quadrotor concept motions description

\section{CONVENTIONAL PD CONTROLLER}

Proportional-plus-derivative(PD) controllers are widely used in the industry $[2,3]$. The main reason is its relatively simple structure, which can be easily understood and implemented in practice [4]. The widespread use of PD-type controllers in industries has affected efforts in the design and tuning of conventional PD controllers so as to achieve an optimal performance for the control system [5] The quadrotor in nature is very unstable. In order to stabilize the quadrotor, PD controller is needed to be developed within the system. PD controller is the most widely used controller because of its simplicity and robustness. The mathematical equivalent of PD control algorithm can be expressed as

$$
\mu=k_{p} e+k_{d} \frac{d e}{d t}
$$

Where $\mathrm{k}_{\mathrm{p}}$ and $\mathrm{k}_{\mathrm{d}}$ are the proportional, and derivative gains respectively. To achieve stabilization, three PD controllers are implemented for three different axes: roll, pitch and yaw respectively. For a sensor feedback, we use gyro sensor which is three axes angular rate sensor. 


\section{IMPLEMENTATION OF SIMULINK}

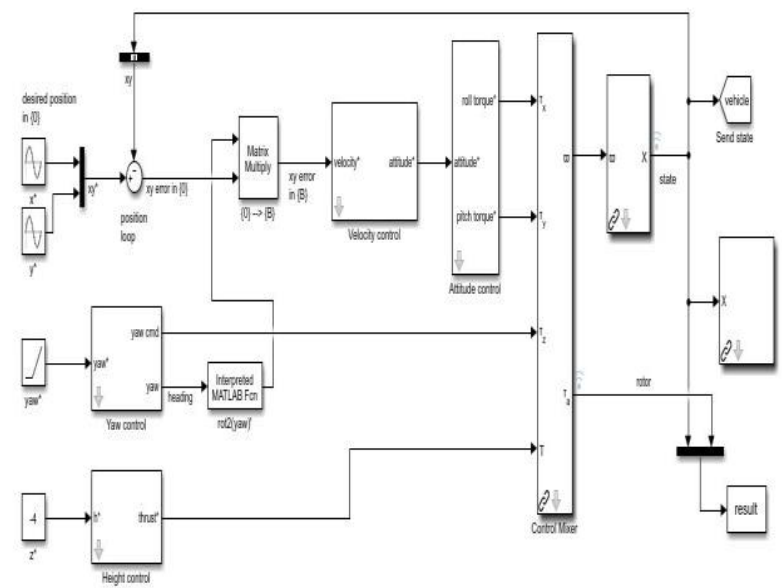

Figure 2. Block Diagram of Simulation

This figure contains yaw control, high control, velocity control, attitude control. Firstly, the velocity control of proportional gain is 0.1 and derivative gain is 2 . The High control of proportional gain is 4 and derivative gain is 1 . The altitude control of proportional gain is 100 and derivative gain is 1 . The yaw control of proportional gain is 20 and derivative gain is 2 . The simulation result of desired value and actual value as shown in figure 4 . The error value $\mathrm{x}, \mathrm{y}$ and yaw as shown in figure 5. And then, this velocity, high, altitude and yaw control with proportional gain is changing and the derivative is not changing this result of desired and actual value as shown in figure 7, and the error value of $x, y$ and yaw as shown in figure 8 .

\section{SIMULATION RESULT}

The simulation result is the compared of desired and actual value and error without changing $\mathrm{P}$ and with changing $\mathrm{P}$. The changing of $\mathrm{P}$ is high the error is high. The greater the value of $\mathrm{P}$, the higher the error becomes. This paper states the result of this desired and actual error of $\mathrm{X}, \mathrm{Y}$ and Yaw.

Table 1. PD gain values for without changing $P$

\begin{tabular}{|c|c|c|}
\hline Type & $\mathbf{k}_{\mathbf{p}}$ & $\mathbf{k}_{\mathbf{d}}$ \\
\hline Velocity Control & 0.1 & 2 \\
\hline High Control & 4 & 1 \\
\hline Altitude Control & 100 & 1 \\
\hline Yaw Control & 20 & 2 \\
\hline
\end{tabular}

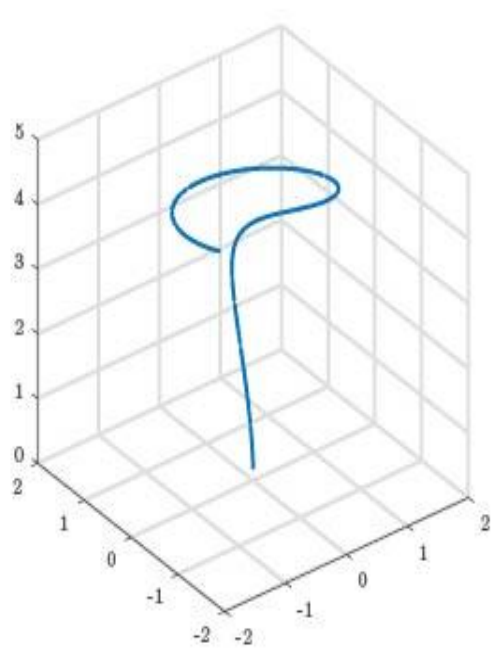

Figure 3. The trajectory of UAV without changing $P$
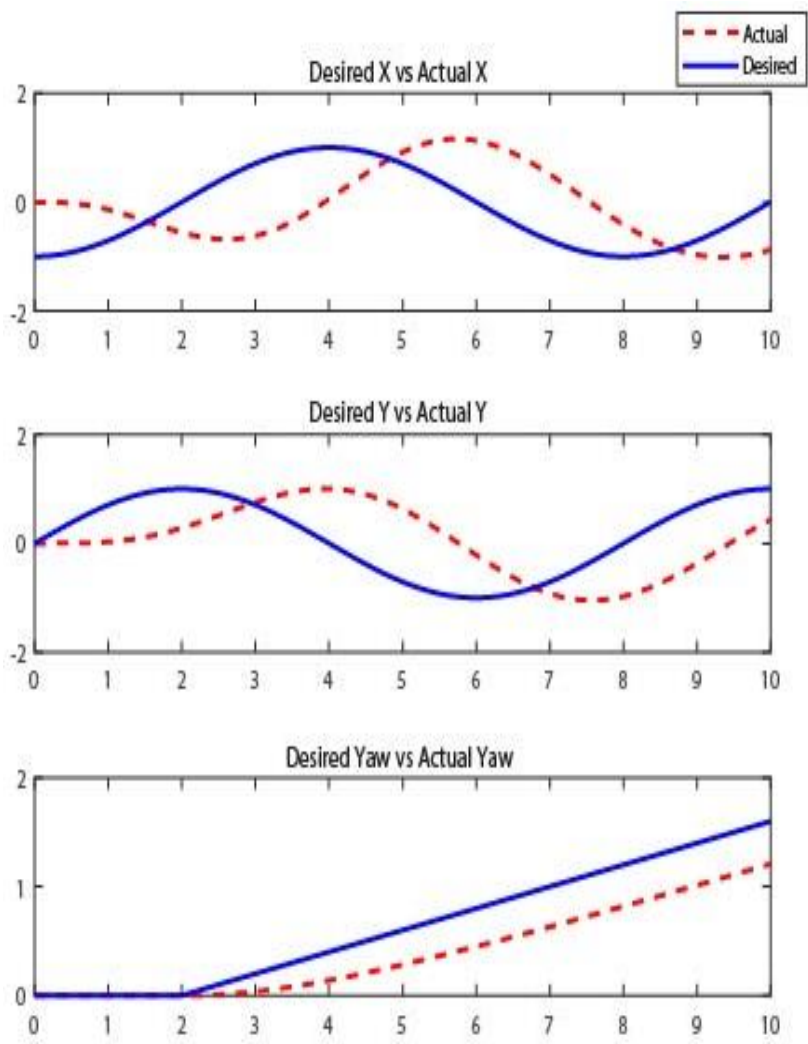

Figure 4. Plot of desired \& actual value of $X, Y \&$ Yaw 

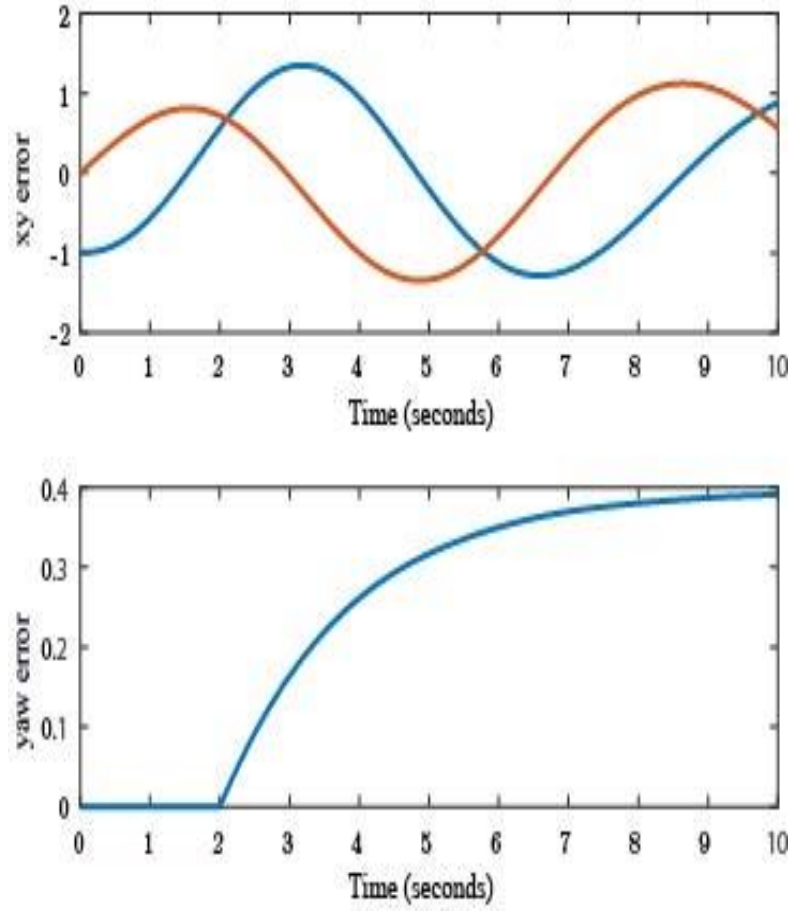

Figure 5. Plot of error in $X, Y \&$ Yaw without changing $P$

Table 2. PD gain values for with changing $P$

\begin{tabular}{|c|c|c|}
\hline Type & $\mathbf{k}_{\mathbf{p}}$ & $\mathbf{k}_{\mathbf{d}}$ \\
\hline Velocity Control & 0.2 & 2 \\
\hline High Control & 8 & 1 \\
\hline Altitude Control & 200 & 1 \\
\hline Yaw Control & 40 & 2 \\
\hline
\end{tabular}

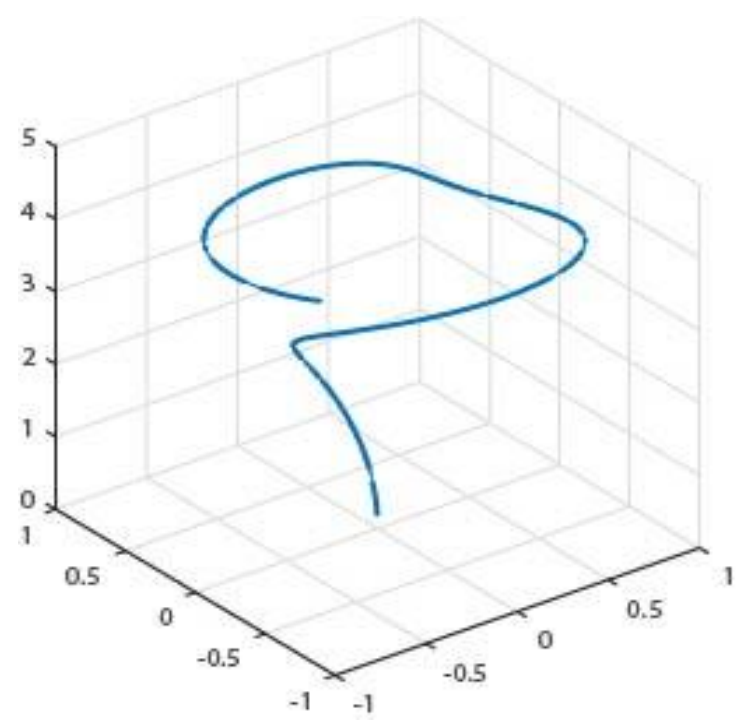

Figure 6. The trajectory of UAV with changing $P$
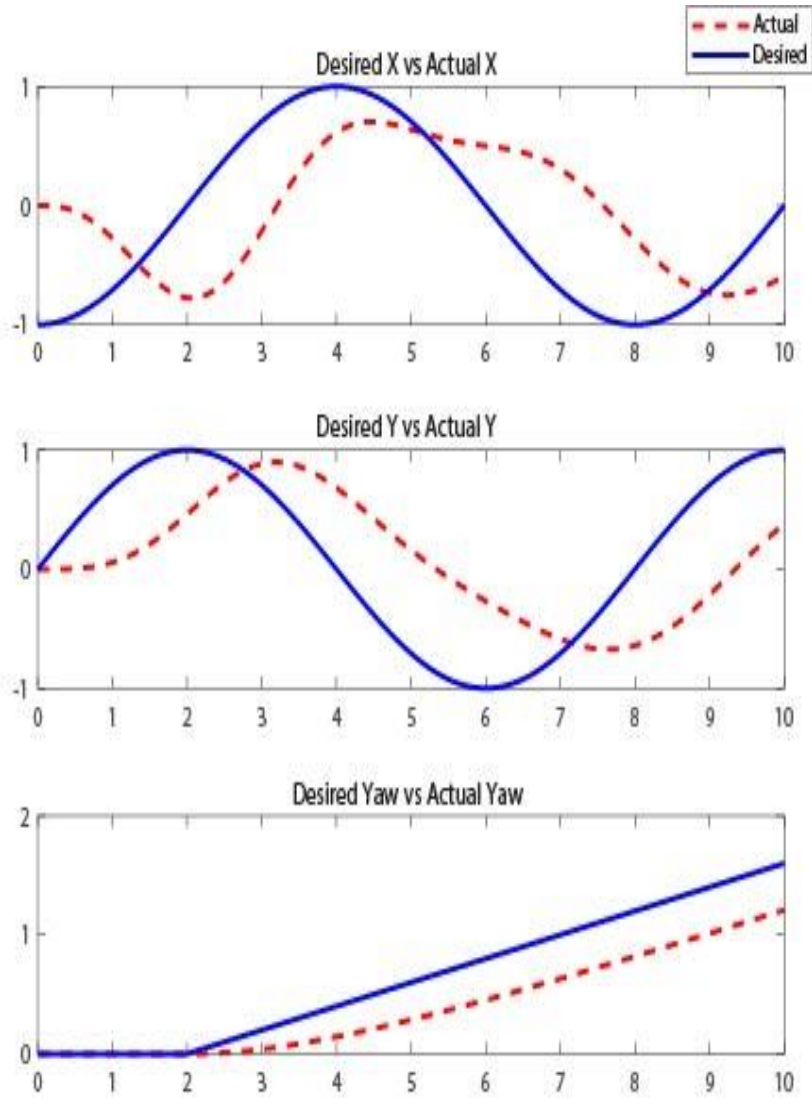

Figure 7. Plot of desired \& actual value of X, Y \& Yaw
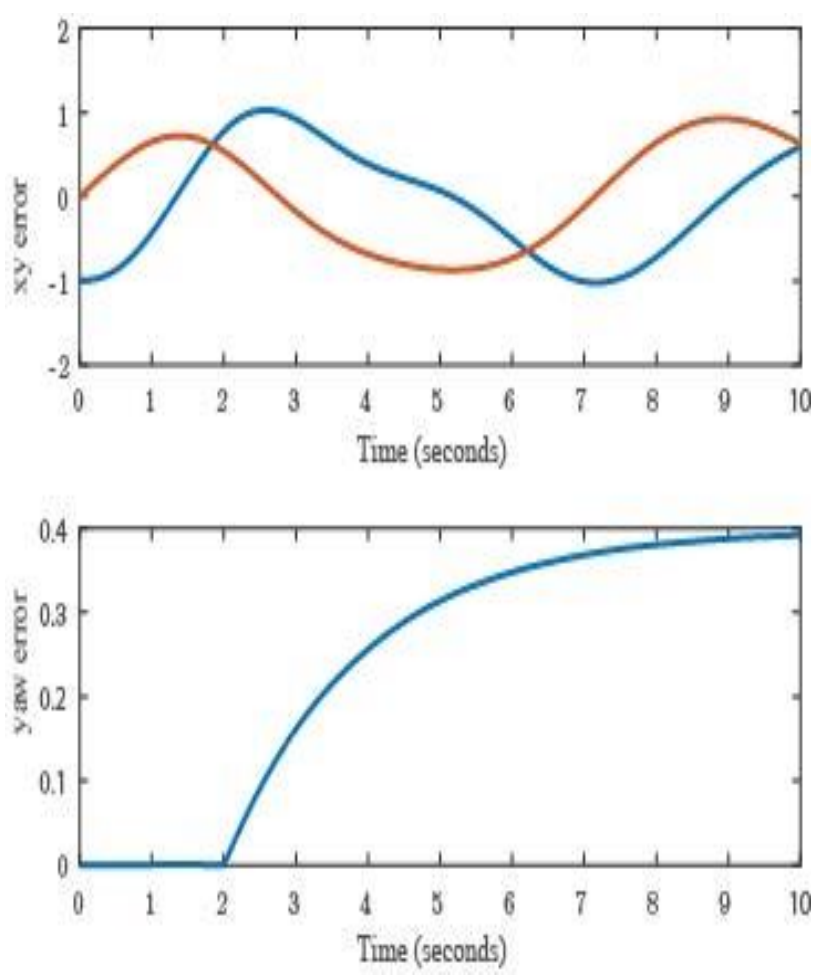

Figure 8. Plot of error in $X, Y \&$ Yaw with changing $P$ 


\section{CONCLUSION}

Unmanned aerial vehicles (UAV) can provide a critical support for search and rescue operations. However, in order to achieve their full potential, it is necessary to properly account for all the parameters that can affect the flight of the UAVs such as quality of sensory operations (that can depend on the position of the UAVs for instance), energy constraints, environmental hazards or data sharing constraints between UAVs and rescue teams.

This paper presented the design of a PD controller algorithm to control the quadrotor system. The simulation result of the desired value and actual value of $\mathrm{X}, \mathrm{Y}$ and Yaw compared without changing PD and with changing PD controller.Also the compared of this result can be improved by using others features.

\section{ACKNOWLEDGMENTS}

I greatly appreciate to all teachers from the Department of Electronics Engineering, Technological University (Thanlyin) who work in Research.

I would like to give great thanks to Research Department of Electronics Engineering, Technological University (Thanlyin) for kind permission to study related on-going research projects.

\section{REFERENCES}

[1] Comparison of Backstepping, Fuzzy-PID, and PID Control Techniques using $\mathrm{X} 8$ model in relation to $\mathrm{A}^{*}$ Path Planning Yi Zhou Singapore Institute of Technology, Singapore.

[2] K. J. Astrom and T. Hagglund, "Automatic Tuning of Regulators with Specifications on Phase and Am- plitude Margins," Journal of Automatica, Vol. 20, No. 5, 1984, pp. 645-651. doi:10.1016/0005-1098(84)90014-1.

[3] W. K. Ho, O. P. Gan, E. B Tay and E. L. Ang, "Performance and Gain and Phase Margins of Well-Known PID Tuning Formulas," IEEE Transactions on Control System Technology.

[4] Q. G. Wang, T. H. Lee, H. W. Fung, Q. Bi and Y. Zhang, "PID Tuning for Improved Performance," IEEE Transactions on Control Systems Technology.

[5] Y. C. Cheng and C. Hwang, "Stabilization of Unstable First-Order Time-Delay," Journal of the Chinese Institute of Engineers.

[6] D. Vrancic, S. Strmenik, J. Kocijan and P. B. M. Oliveira, "Improving Disturbance Rejection of PID Controllers by Means of the Magnitude Optimum Method," ISA Trans- actions. 